\title{
Àngel Guimerà en el cine japonés: intertextualidad intercultural en la adaptación de Kenji Mizoguchi del drama Terra Baixa
}

\section{Àngel Guimerà in Japanese Film: Cross-Cultural Intertextuality in Kenji Mizoguchi's Adaptation of the play Terra Baixa}

\author{
Alex PINAR \\ Aichi Prefectural University (Nagakute, Japón) \\ alex.pinar@yahoo.com \\ ORCID ID: 0000-0003-2780-9099
}

\begin{abstract}
Resumen: Desde los inicios de la industria cinematográfica en Japón se realizaron un gran número de películas basadas en obras literarias occidentales, especialmente durante las décadas de 1910 y 1920. El director que realizó el mayor número de estas adaptaciones fue Kenji Mizoguchi. Entre estas se encuentra Jinkyō (Este mundo polvoriento, 1924), basada en del drama de Àngel Guimerà Terra baixa (Tierra baja). Dado que la película está perdida, en este estudio se analiza esta adaptación intercultural a través de fotogramas y reseñas de revistas de cine publicadas en la época. Se describe cómo y en qué momento llegó a ese país la obra de Guimerà, cómo el cineasta la trasladó al contexto cultural japonés, y de qué modo influyeron el contexto histórico-social y la tradición cultural en el proceso de adaptación.
\end{abstract}

Palabras clave: intertextualidad, adaptación intercultural, Kenji Mizoguchi, Àngel Guimerà, teatro catalán, cine japonés.

\begin{abstract}
At the dawn of Japanese cinema, especially in the 1910 s and 1920 s, many movies were adapted from the Western novels. Kenji Mizoguchi made several of such adaptations, including his 1924 film Jinkyō (This Dusty World), adapted from Terra baixa (Martha of the Lowlands), a drama by Àngel Guimerà. While the film in question has been lost, its stills and reviews of film magazines published at the time of screening have been archived. This paper draws on such materials and analyzes intercultural aspects of Mizoguchi's adaptation. It provides a brief overview on the reception of Guimerà's work. It also analyzes the process through which Mizoguchi adapted the play to the Japanese context. Finally, it offers insights on how the historical-social context and the cultural tradition influenced the director's adaptation process.
\end{abstract}

Key Words: intertextuality, intercultural adaptation, Kenji Mizoguchi, Àngel Guimerà, Catalan theater, Japanese cinema. 


\section{INTRODUCCIÓN}

La adaptación cinematográfica se puede definir como un proceso dialógico intertextual en el que el cineasta crea una nueva obra artística (hipertexto) a través de la transformación de diversos elementos narrativos de una obra literaria (hipotexto) (Stam, 2000: 68). En el caso de películas basadas en literatura de una esfera cultural diferente, la adaptación puede implicar también la modificación de los códigos culturales de la obra literaria. Probablemente, el ejemplo más conocido de este tipo de adaptaciones, denominadas «cine intercultural» por autores como Goodwin (1994: 1), serían las películas de samuráis de Akita Kurosawa Kumonosu-jō (Trono de sangre, 1957) y Ran (1985), la cuales estaban basadas en las obras teatrales de Shakespeare Macbeth y King Lear (El rey Lear) respectivamente.

La adaptación intercultural es un fenómeno que se inició en Japón décadas antes de que los filmes de Kurosawa tuvieran reconocimiento mundial y fueran exhibidos en festivales de cine internacionales. Desde los inicios de la industria cinematográfica en ese país se realizaron numerosos cortometrajes y películas basados en obras literarias occidentales, especialmente durante las décadas de 1910 y 1920. El director que realizó el mayor número de adaptaciones de literatura occidental durante toda su carrera, mayoritariamente durante los años 20, fue Kenji Mizoguchi, considerado uno de los directores de cine más importante en los anales del cine japonés gracias a sus obras maestras Gion no shimai (Las hermanas de Gion, 1936), Naniwa eregii (Elegía de Naniwa, 1936), Ugetsu Monogatari (Cuentos de la luna pálida, 1953), o Sanshō Dayū (El intendente Sansho, 1954).

Además de dirigir películas basadas en obras de literatura anglosajona, alemana, francesa o rusa, Mizoguchi también filmó en los años 20 una adaptación de la obra de teatro de Àngel Guimerà Terra baixa (Tierra baja, 1896), titulada Jinkyō (Este mundo polvoriento, 1924) ${ }^{1}$. Puesto que esta película está hoy perdida (al igual que todas las adaptaciones de literatura occidental que hizo Mizoguchi en los años 20), y dado que la literatura en lengua catalana era prácticamente desconocida en Japón en esa época, es inevitable preguntarse cómo y en qué momento llegó a ese país la obra de Guimerà, cómo Mizoguchi adaptó este drama ambientado en la Cataluña rural al contexto cultural japonés, y de qué modo influyeron el contexto histórico-social y la tradición cultural en el proceso de adaptación.

Aunque la película se perdió, probablemente durante los frecuentes bombardeos llevados a cabo durante la Segunda Guerra Mundial, sí que se han conservado fotogramas y reseñas aparecidas en revistas de cine de la época que permiten realizar un examen comparativo entre la película y la

\footnotetext{
${ }^{1}$ El título de la película es conocido también en español como El mundo de aquí abajo. Consideramos que la traducción más adecuada sería Este mundo polvoriento ya que mantiene el sentido simbólico del título original en japonés.
} 


\section{Àngel Guimerà en el cine japonés}

obra de Guimerà ${ }^{2}$. De este modo, siguiendo el enfoque dialógico intertextual de Stam (2000), se analizan las permutaciones en elementos narratológicos (localización de la acción, argumento y personajes) y se describen la transformación de los códigos culturales con el objetivo de dar respuesta a las preguntas planteadas previamente.

\section{AdAPTACIONES DE literatura OCCIDENTAL EN JAPÓN EN LAS DÉCADAS DE 1910 Y 1920}

El cine llegó a Japón poco tiempo después de su invención, en 1897. Durante los primeros años fue frecuente el rodaje y exhibición de breves escenas de la vida en las calles de Tokio, de danzas de geisha, o de secuencias con conocidos pasajes de kabuki. Posteriormente se empezó a filmar películas jidai-geki (películas de época ambientadas en la época de Edo) basadas en obras de kabuki, y películas gendai-geki (ambientadas en un contexto moderno), inspiradas en su mayoría en escenas de teatro shinpa (nueva escuela). Este tipo de teatro apareció en la década de 1880 como reemplazo al kabuki, cuya temática y estilo no permitían mostrar las inquietudes del Japón moderno. Tanto las películas gendai-geki como las obras de teatro shinpa trataban temas contemporáneos con un estilo realista y melodramático, aunque todavía compartían algunas características del teatro kabuki como los manierismos en la actuación y la presencia de oyama, es decir, actores masculinos que interpretaban personajes femeninos ${ }^{3}$.

En la década de 1910 los estudios empezaron a producir también películas basadas en obras literarias occidentales, las cuales habían sido previamente representadas en los teatros shingeki (nuevo drama). Este tipo de teatro, iniciado a principios del siglo XX, tenía un carácter intelectual y vanguardista (Satō, 2008: 20). En sus salas se escenificaban obras de destacados autores occidentales como O'Neill, Ibsen, Chéjov, Gorki o Tolstoi, y también obras de escritores japoneses como Kaoru Osanai. Estas adaptaciones, como Kachusha (Resurrección, 1914) de Kiyomatsu Hosoyama (basada en la novela Resurrección de Tolstoi), o Ikeru shikabane (El cadáver viviente, 1918) de Eizō Tanaka (basada en la obra teatral homónima de Tolstoi), estaban dirigidas a un público educado y familiarizado con la cultura literatura occidental. Intentaban ser «fieles al original» $y$ reproducir, como en un escenario de teatro shingeki, el contexto cultural

${ }^{2}$ En la National Diet Library de Tokio (el equivalente japonés a la Biblioteca Nacional) se pueden consultar revistas de cine publicadas aquellos años. En el National Film Center (también situado en Tokio) se conservan también fotogramas de películas perdidas. Tanto la reseña como los fotogramas utilizados para realizar esta investigación se han encontrado en ambas instituciones.

3 Los actores oyama, también conocidos como onnagata, asumieron papeles femeninos en el teatro kabuki desde 1629, cuando el Shogunato emitió un decreto que prohibía a todas las actrices subir a los escenarios al considerar que su presencia tenía un efecto negativo en la moral pública. 
de la obra a través de la escenografía, la puesta en escena y la ambientación.

En los años 20 la occidentalización y el modernismo caracterizaron la vida cultural de las grandes ciudades y el cine se convirtió rápidamente en el entretenimiento principal de las masas urbanas. En esos años, en los que se establecieron las normas estéticas y se fijaron los modos de producción, distribución y exhibición (Wada-Marciano, 2008: 2), surgieron nuevas compañías como Shochiku Kinema y Taikatsu Co., las cuales tenían como objetivo producir películas siguiendo las técnicas cinematográficas y los estilos narrativos del cine europeo y norteamericano. Progresivamente se abandonaron técnicas y convenciones teatrales que habían perdurado en años anteriores: se dejó de usar actores oyama, se introdujeron técnicas narrativas como el uso flashbacks, y se generalizó el uso de intertítulos, minimizando la importancia y presencia de los narradores en vivo llamados benshi (Anderson, 1992: 273). El cine teatralizado, llamado también cine «enlatado» (Komatsu, 1992: 231), quedó obsoleto a medida que los nuevos estudios producían filmes rodados con técnicas y estilos similares a las películas americanas y europeas, las cuales copaban el 75\% del mercado japonés (Gerow, 2008: 8).

La primera película basada en una obra literaria occidental realizada siguiendo un estilo cinematográfico no teatral y adaptando la historia al contexto cultural japonés fue Rojō no reikon (Almas en el camino, 1921), dirigida por Minoru Murata en cooperación con el director de teatro shingeki y escritor Kaoru Osanai. Rojō no reikon, basada en la obra Na dnié (Los bajos fondos) de Gorki y en el drama Mutter Landstrasse (Niños de la calle) de Wilhelm Schmidtbonn, fue aclamada debido a sus innovaciones técnicas y a la forma en que se retrataban los infortunios de los personajes, con los cuales los espectadores japoneses se podían sentir identificados (Richie, 2012: 39). Una de las consecuencias del éxito de este filme fue que los estudios y directores perdieron el interés por producir adaptaciones que fueran «fieles al original» y que reprodujeran los códigos culturales de la obra literaria. A partir de ese momento las películas basadas en la literatura occidental se realizaron siguiendo procesos interculturales e intertextuales en los que las obras literarias se modificaron libremente, adaptándolas al contexto cultural japonés y haciéndolas más atractivas para el público medio urbano.

Como hemos mencionado en la introducción, el cineasta que realizó el mayor número de adaptaciones de literatura occidental durante las décadas de 1920 y 1930 fue Kenji Mizoguchi, quien antes de dirigir su primera película había trabajado como asistente de Eizō Tanaka. Tras debutar como director en 1922 con la película Ai ni yomigaeru hi (El día en el que regresó el amor), Mizoguchi dirigió antes de acabar la década ocho películas basadas en obras de literatura occidental. Además de Jinkyō, los filmes que tuvieron más repercusión, tal como muestran las reseñas y críticas encontradas en revistas de la época, fueron 813-Rupimono (813: Las 


\section{Àngel Guimerà en el cine japonés}

aventuras de Arsène Lupin, 1923), basada en la novela de Maurice Leblanc 813; Kiri no minato (El puente de la niebla, 1923), una adaptación de la obra teatral de Eugene O'Neill Anna Christie; y Chi to rei (La sangre y el alma, 1923), película expresionista inspirada en el filme de Robert Wiene Das Cabinet des Dr. Caligari (El gabinete del doctor Caligari, 1920) y basada en la novela corta de E.T.A. Hoffmann Mademoiselle de Scudéri (La Señorita de Scuderi).

\section{JINKYŌ (ESTE MUNDO POLVORIENTO, 1924)}

Poco tiempo después de su aparición, el drama de Àngel Guimerà Terra baixa fue traducido al castellano por José Echegaray. A partir de la traducción española la obra se difundió rápida y exitosamente a otros países, publicándose tanto traducciones como versiones adaptadas al contexto cultural de cada país (Gallén, 2012: 217). También el drama de Guimerà fue la base de las óperas Tiefland (1903), escrita por Eugen d'Albert a partir de un libreto de Rudolph Lothar, y La catalane (1907), musicalizada por Fernand Le Borne con libreto escrito por Paul Ferrier y Louis Tiercelin. Asimismo, pronto se realizaron adaptaciones cinematográficas de la obra original y de sus versiones: en 1907 Fractuós Gelabert filmó el cortometraje de 20 minutos Tierra baja; en Argentina se estrenó en 1913 una versión de la obra con el mismo título, dirigida por Mario Gallo; el año siguiente se produjo una producción estadounidense titulada Martha of the Lowlands (Marta de las Tierras Bajas) dirigida por J. Searle Dawley; y una adaptación alemana titulada Tiefland (Tierras bajas) fue filmada en 1922 por Adolf Edgard Licho.

En Japón también se publicó una versión de la obra titulada Jinkyō (Este mundo polvoriento) escrita por Kaoru Osanai en 1916. Este drama no se basó en el original en catalán o en la traducción de Echegaray, sino que se inspiró, tal como afirma Osanai al final de su obra, en el libreto de la ópera Tiefland de Eugen d'Albert. Antes de ser llevada a la pantalla, Jinkyō fue representada por primera vez en el teatro Yuraku-za en el año 1922. Este montaje estaba dirigido por el mismo Osanai e interpretado por el grupo teatral Shingeki-za (Compañía de nuevo teatro), establecido en 1919 con la voluntad de representar obras occidentales (Poulton, 2010: 31). Tanto la ópera de d'Albert como la versión de Osanai mantenían la trama argumental de la obra de Guimerà y casi el mismo número de personajes, aunque en el texto de Osanai estos tenían nombres japoneses y la historia estaba ambientada en Japón, en un área rural de la región montañosa de Tōhoku.

El guion de la película, escrito por Sōichiro Tanaka, se basó a su vez en la versión de la obra escrita por Osanai. La trama resumida por el crítico de cine Roppa Furukawa en la revista Kinema Junpō (1924: 10) muestra que el argumento de la película, desarrollado también en un área rural de Japón, era similar al de la obra de Guimerà y de las versiones de Lothar y Osanai (nos referiremos a los tres textos como «los hipotextos» a 
partir de ahora). De este modo, el filme desarrollaba la historia de un terrateniente arruinado llamado Senkichi, quien se propone casarse con una mujer rica y usar su dinero para pagar sus deudas. Sin embargo, desea mantener su relación con su amante, Omatsu. Para evitar sospechas la obliga a casarse con otro hombre, un leñador llamado Rokuzō que vive aislado en las montañas. Una vez casados Rokuzō se da cuenta de que la boda fue una farsa y anuncia su intención de regresar a las montañas. Omatsu decide en ese momento seguirlo y dejar las tierras de Senkichi. Para impedirlo el terrateniente lucha con Rokuzō, quien termina estrangulándolo:

Un terrateniente de montaña llamado Nomura Senkichi estaba profundamente endeudado debido a los fracasos de sus negocios y por ese motivo decidió casarse con una mujer rica para obtener su dote. Sin embargo, Senkichi tenía una amante llamada Omatsu, una artista itinerante que llegó a su casa tiempo atrás. Decidió obligarla a casarse con otro hombre por el bien de las apariencias, sin dejar de mantenerla como su amante. Senkichi eligió a un hombre leñador llamado Rokuzō para casarse con Omatsu. Él estaba encantado de bajar de las montañas el día de la boda. Omatsu, en realidad, tenía miedo de Rokuzō y se enfadó con Senkichi por entregarla a este hombre de aspecto rudo. Omatsu lloró y Rokuzō se sorprendió. Al descubrir la verdad de la situación, Rokuzō decidió abandonar este «mundo sucio» y regresar a la montaña. Omatsu encontró la reacción de Rokuzō noble y lo siguió. Senkichi se sorprendió de que Omatsu hubiera seguido a Rokuzō, por lo que los persiguió. Después de una feroz lucha, Rokuzō estranguló a Senkichi hasta la muerte. Rokuzō dijo: «Mi querida Omatsu, volvamos a nuestra montaña», y desaparecieron (1924: 10) ${ }^{4}$.

Aunque la película desarrollaba una trama similar a la de los hipotextos, esta era protagonizada por seis personajes en lugar de doce como en la obra de Guimerà o de diez como en el en el libreto de Lothar y la versión de Osanai. Como personajes secundarios aparecían Orie, una niña amiga de la protagonista; Gonji, un guardia de molino de agua; y Soncho, el jefe del pueblo (rol similar al del siniestro capellán que aparece en la obra de Guimerà). Como protagonistas aparecían los ya mencionados Senkichi, el hacendado sin escrúpulos que gobierna la vida de sus campesinos, considerándolos de su propiedad, y Omatsu y Rokuzō, la amante del terrateniente y el leñador solitario. Los personajes de Omatsu y Rokuzō presentaban personalidades y funciones narrativas similares a los protagonistas de los hipotextos. Sin embargo, el guionista introdujo algunas modificaciones en su caracterización para adaptar la historia al contexto cultural japonés, de modo fuera más verosímil y comprensible para el espectador medio.

\footnotetext{
${ }^{4}$ Reseña traducida por el autor con la colaboración de Yuta Yasuda.
} 


\section{Àngel Guimerà en el cine japonés}

En los hipotextos la protagonista vive toda su vida en extrema pobreza hasta que un día, vagabundeando con un mendigo que la cuida, llega a casa del terrateniente. Con el tiempo ella se convierte en su amante, dejando atrás sus penurias. En cambio, en la película, tal como se afirma en la reseña de Furukawa, Omatsu es una artista itinerante. Por tanto, debido a su profesión ella pertenece al grupo social de los hinin. En este grupo se incluían personas que tenían ocupaciones consideradas impuras según las creencias budistas o sintoístas, tal como mendigos, prostitutas y artistas itinerantes como Omatsu. Los hinin, junto con los eta (grupo al que pertenecían curtidores, carniceros o verdugos, entre otras profesiones que eran consideradas extremadamente impuras), formaban la casta de los burakumin, cuyos miembros, ya desde la era medieval, eran considerados «no humanos» y eran víctimas del ostracismo y de la discriminación. Era imposible para un miembro del grupo eta cambiar de casta, pero sí se permitía que los hinin lo hicieran (Abe, 2003: 70).

Interpretamos que en el filme ella conoce a Senkichi en uno de sus desplazamientos y que, obligada por la miseria, acepta su invitación a quedarse con él e iniciar una relación extramatrimonial. Dado que la marginación de los burakumin continuaba presente en el año en el que se rodó la película, a pesar de la abolición legal de ese estatus social durante la era Meiji (1868-1912) y de los intentos de erradicar las actitudes de rechazo hacia ese colectivo llevados a cabo durante la década de 1920 (Ishikida, 2005), el concubinato con Senkichi le ofrece a Omatsu la oportunidad de abandonar su casta y, al igual que la protagonista de los hipotextos, ascender socialmente a un estatus superior.

El personaje de Rokuzō comparte también muchas similitudes con el protagonista de los hipotextos. Son hombres ingenuos, rudos y solitarios que viven y trabajan solos en las montañas y que descienden a las llanuras para casarse engañados por el terrateniente. En los hipotextos, el protagonista es un pastor, cuya imagen está vinculada al lobo, animal percibido en la tradición cultural occidental como una amenaza para los rebaños y para los medios de vida humanos, representado en la tradición literaria escrita y oral como una metáfora de la avaricia, la violencia y el mal. Este animal deviene el leitmotiv de los hipotextos: el pastor lucha con el lobo con sus propias manos dándole muerte, al igual que hace con el terrateniente, al que se caracteriza metafóricamente como un lobo del cual el pastor tiene que proteger a la protagonista. En cambio, en la película Rokuzō es leñador. Al cambiarse la profesión del protagonista se difumina la presencia del lobo y su simbología. Como puede verse en la fotografía del rodaje (fig. 3), este animal es sustituido en el filme por un oso al que el protagonista mata en una lucha cuerpo a cuerpo, tal como acaba haciendo con el hacendado, quien sería comparado simbólicamente como un oso del que Rokuzō defiende a la protagonista.

Existen razones culturales que explican este cambio en la profesión del protagonista masculino y la sustitución del lobo por el oso como leitmotiv 
de la historia. Por un lado, habría sido inverosímil ver en pantalla a un personaje japonés trabajando como pastor, ya que esta era una profesión muy poco común en Japón en ese momento, a pesar de los esfuerzos fallidos del gobierno Meiji para alentar y promover la cría de ovejas con la finalidad de que Japón pudiera ser autosuficiente en lana. Por otro lado, dejando de lado el hecho de que los lobos se extinguieron en Japón en el siglo XIX, los espectadores japoneses no habrían entendido el simbolismo implícito en la presencia del lobo y su muerte a manos de Rokuzō, ya que el lobo es considerado como un mensajero de los dioses en las creencias sintoístas.

La mitología japonesa presenta al lobo como un animal favorable. Lejos de ser visto como una amenaza para la vida de los aldeanos, el lobo es percibido como una criatura que los protege a los humanos de los animales salvajes que amenazan sus granjas y campos (Knight, 1997: 140). Asimismo, existe en el folclore japonés la creencia en el okuriōkami (lobo escolta), animal que sigue a los humanos que caminan solos a través de un bosque hasta que llegan a su destino sin hacerles ningún daño (Knight, 1997: 136). Según la tradición, una persona que matara a un lobo podría exponer a sí mismo y a su familia al riesgo de represalias espirituales. En lugar del lobo, en Japón son los osos y otros animales salvajes los que son percibidos como un peligro. El folclore japonés habla de un monstruo mitológico llamado onikuma (oso demonio), que ocasionalmente emerge de los bosques para buscar comida, y que reacciona agresivamente si se encuentra con los seres humanos (Komatsu, 2017: 96), tal como ocurre en la película.

Respecto a los espacios en los que transcurre la acción, ya hemos comentado que tanto en la versión del drama de Osanai como en el filme la historia trascurre en una zona rural y montañosa de Japón. Como en los hipotextos, el protagonista vive en las tierras altas y desciende a las llanuras para contraer matrimonio. Ambos espacios están cargados de un marcado simbolismo tanto en las distintas versiones del drama de Guimerà como en la película de Mizoguchi. Las tierras altas son una zona que representa la libertad, la pureza, la honestidad y todo lo que es bueno por naturaleza. En contraste, las tierras bajas son un lugar materialista y pernicioso que representa la miseria, los prejuicios y los males de la humanidad. Esta dicotomía entre las tierras altas y las tierras bajas y su simbolismo se refleja también en el título original de la película, Jinkyō (塵境), el cual tiene un profundo significado alegórico. Está formado por los ideogramas Jin (塵) y Kyō (境). Jin denota polvo y suciedad. En los textos budistas representa además la profanación, la impureza y la aflicción. Kyō significa borde o límite, pero también un área o territorio. En los textos budistas, Kyō representa asimismo un estado mental insano. En consecuencia, el título alude de manera metafórica al lugar el que se desarrollan los acontecimientos, es decir, al «mundo polvoriento» de las 


\section{Àngel Guimerà en el cine japonés}

tierras bajas, un espacio fronterizo entre el bien y el mal, entre la pureza de la vida en las montañas y la degradación moral de las llanuras.

\section{CONCLUSIONES}

Como hemos descrito, la obra Terra baixa llegó a Japón a través del libreto de la ópera de d'Albert, el cual fue adaptado a su vez al contexto japonés por Kaoru Osanai. En su versión, Osanai mantuvo el argumento de la obra y la mayoría de los personajes, situando la acción en una zona rural del área de Tōhoku.

A través del análisis de fotogramas y de una reseña aparecidos en revistas de cine de la época, hemos podido comprobar que en la película realizada por Mizoguchi se mantuvo también la trama de la historia. Sin embargo, la obra se adaptó modificando algunos códigos culturales en concordancia con la realidad histórico-social y la tradición cultural japonesa, haciendo que la película fuera más comprensible para el público general. Estos cambios fueron más notables, además de en la localización de la acción, en la caracterización de los personajes principales y en la transformación del leitmotiv de la obra y su simbolismo.

\section{ILUSTRACIONES}

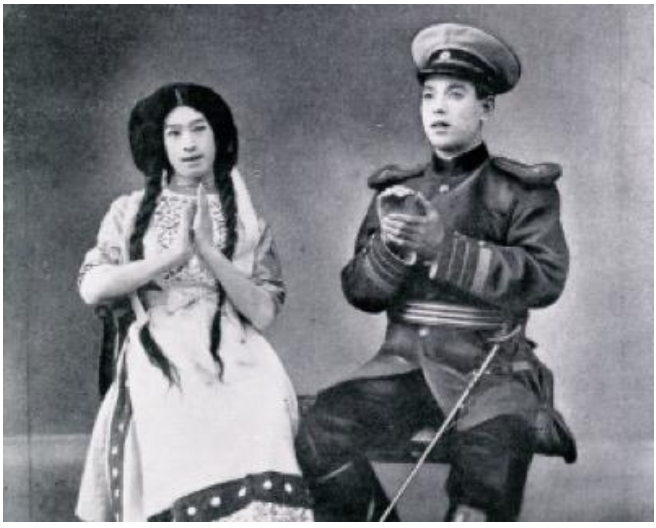

Fig. 1. Fotograma de la película Kachusha de Hosoyama (fuente: https://commons.wikimedia.org/wiki/File:Katyusha_1914_fi $\operatorname{lm} 1 . j p g)$. A la izquierda, el actor Teijirō Tachibana representando el papel de Katiusha Máslova. A la derecha, Sekine Tappatsu intepretando al prícipe Nejliúdov. Los actores visten ropas de estilo ruso con el objetivo de aportar más realismo y de reproducir el contexto cultural de la obra literaria. Nótese que en las adaptaciones realizadas en la década de 1910 todavía se utilizaban actores oyama. 


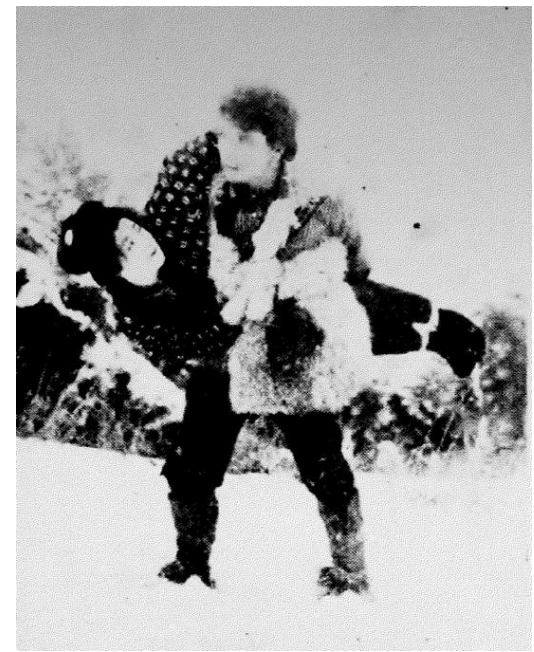

Fig. 2. Fotograma de la escena final en la que Rokuzō regresa a las montañas llevando con él a Omatsu (publicado en la revista Kinema Junpō, 160, 1924, pág. 18).

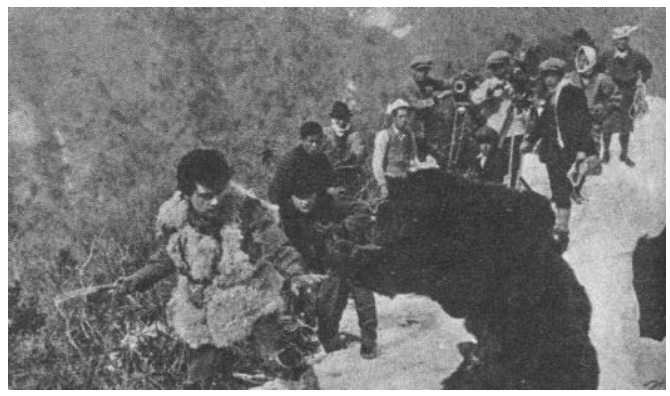

Fig. 3. Fotografía del rodaje de la película en la que Rokuzō lucha contra un oso en lugar de un lobo, tal como ocurre en la obra de Guimerà, en la ópera d'Albert y en la versión de Osanai (publicada en Keiichi Kondō [ed.], Eiga sutā zenshū [Guía completa de las estrellas de cine], vol.1., Tokio, Heibon sha, 1929, pág. 67). 


\section{Bibliografía CITADA}

ABE, Chikara (2003), Impurity and Death: A Japanese Perspective, Boca Raton, Universal Publishers.

Anderson, Joseph (1992), «Spoken Silent in the Japanese Cinema; or, Talking to Pictures: Essaying the Katsuben, Contextualizing the Texts», en A. Nolletti y D. Desser (eds.), Reframing Japanese Cinema: Authorship, Genre, History, Bloomington, Indiana University Press, págs. 259-310.

FURUKAWA, Roppa (1924), «Jinkyō», Kinema Junpō, 160, pág.10.

GALLÉN, Enric (2012), «Guimerà a Europa i Amèrica», Catalan Historical Review, 5 , págs. 85-100.

Gerow, Aaron (2008), A Page of Madness: Cinema and Modernity in 1920s Japan, Michigan, Center for Japanese Studies, The University of Michigan Press.

Goodwin, James (1994), Akira Kurosawa and Intertextual Cinema, Baltimore, Johns Hopkins University Press.

GuimerÀ, Àngel (1897), Terra Baixa, Barcelona, Butxaca 62.

IsHIKIDA, Miki (2005), Living Together: Minority People and Disadvantaged Groups in Japan, Bloomington, iUniverse.

KNIGHT, John (1997), «On the extinction of the Japanese wolf», Asian Folklore Studies, 56, págs. 129-159.

Komatsu, Hiroshi (1992), «Some Characteristics of Japanese Cinema before World War I», en Arthur Nolletti y David Desser (eds.), Reframing Japanese Cinema: Authorship, Genre, History, Bloomington, Indiana University Press, págs. 229-258.

Komatsu, Kazuhiko (2017), An Introduction to Yōkai Culture: Monsters, Ghosts, and Outsiders in Japanese History, Tokio, Japan Library.

KONDŌ, Keiichi (1929) (ed.), Eiga sutā zenshū [Guía completa de las estrellas de cine], vol. 1, Tokio, Heibon sha.

LOTHAR, Rudolf (1903), Tiefland (libreto) [En línea: http://kareol.es/obras/tiefland/tiefland.htm. Fecha de consulta: 10/12/2019].

Osanai, Kaoru (1929 [1916]), Jinkyō, en Obras completas. 5, Tokyo, Shin'ya, págs. 586- 635.

Poulton, M. Cody (2010), A Beggar's Art: Scripting Modernity in Japanese Drama, 1900-1930, Honolulu, University of Hawai'i Press.

Richie, Donald (2012), A hundred years of Japanese film: A Concise History, with a Selective Guide to DVDs and Videos, Tokio, Kodansha International.

SATō, Tadao (2008), Mizoguchi and the Art of Japanese Film, Oxford/New York, Berg.

STAm, Robert (2000), «Beyond Fidelity: The Dialogics of Adaptation», en J. Naremore (ed.), Film Adaptation, New Brunswick, Rutgers University Press, págs. 54-76. 
Alex Pinar

Wada-Marciano, Mitsuyo (2008), Nippon modern: Japanese Cinema of the 1920 s and 1930s, Honolulu, University of Hawaii Press.

Fecha de recepción: 15/05/2020.

Fecha de aceptación: 25/07/2020. 